\title{
Pestalotiopsis-Like Species Causing Gray Blight Disease on Camellia sinensis in China
}

Yingjuan Chen, ${ }^{\dagger}$ Liang Zeng, Na Shu, Maoyuan Jiang, Han Wang, Yunjin Huang, and Huarong Tong, ${ }^{\dagger}$ Department of Tea Science, College of Food Science, Southwest University, Chongqing, 400715, China

\begin{abstract}
Gray blight of tea, caused by several Pestalotiopsis-like species, is one of the most destructive foliar diseases in tea cultivation yet the characteristics of these pathogens have not been confirmed until now. With morphological and multigene phylogenetic analyses, we have identified the gray blight fungi as Pseudopestalotiopsis camelliae-sinensis, Neopestalotiopsis clavispora, and Pestalotiopsis camelliae. Phylogenetic analyses derived from the combined internal transcribed spacer, $\beta$-tubulin, and translation elongation factor $1-\alpha$ gene regions successfully resolved most of the Pestalotiopsis-like species used in this study with high bootstrap

supports and revealed three major clusters representing these three species. Differences in colony appearance and conidia morphology (shape, size, septation, color and length of median cells, and length and number of apical and basal appendages) were consistent with the phylogenetic grouping. Pathogenicity tests validated that all three species isolated from tea leaves were causal agents of gray blight disease on tea plant (Camellia sinensis). This is the first description of the characteristics of the three species Pseudopestalotiopsis camelliae-sinensis, N. clavispora, and Pestalotiopsis camelliae as causal agents of tea gray blight disease in China
\end{abstract}

Gray blight, caused by Pestalotiopsis-like species, is one of the most destructive diseases of tea (Camellia sinensis (L.) O. Kuntze) (Muraleedharan and Chen 1997; Sanjay et al. 2008), and has been reported to cause $17 \%$ production loss in southern India (Joshi et al. 2009) and 10 to $20 \%$ yield loss in Japan (Horikawa 1986). Pestalotiopsis is a species-rich asexual genus with appendage-bearing conidia in the Amphisphaeriaceae (Barr 1975, 1990; Kang et al. 1999; Lee et al. 2006). Because of their roles as both an important plant pathogen and a commonly isolated endophyte, Pestalotiopsis spp. have received considerable attention in recent years (Joshi et al. 2009; Wei et al. 2007). Species of Pestalotiopsis are primarily thought of as weak or opportunistic pathogens that may cause little damage to ornamental plants (Pirone 1978). However, recent reports confirmed that Pestalotiopsis spp. are common phytopathogens that can cause a variety of diseases, including leaf spots, gray blight, severe chlorosis, and various postharvest diseases on a wide range of economic crops such as apple, blueberry, coconut, grapevine, guava, hazelnut, lychee, mango, ginger, and tea plant (Crous et al. 2011; Maharachchikumbura et al. 2012, 2013a, b; Zhang et al. 2012a,b, 2013). The number of known Pestalotiopsis spp. is generally increasing, confirming the pathogen's widespread distribution (Hopkins and McQuilken 2000). However, thus far, only several Pestalotiopsis spp. have been reported to occur on C. sinensis. Among them, Pestalotiopsis longiseta has been recorded to cause severe damage to tea production in Japan (Joshi et al. 2009; Takeda 2002) and $P$. camelliae has been reported to cause gray blight disease in China (Chen et al. 2017b).

Pseudopestalotiopsis and Neopestalotiopsis are two novel genera segregated from Pestalotiopsis. The three genera can be easily distinguished on the basis of combined morphological characteristics and DNA sequence analysis of internal transcribed spacer (ITS), partial $\beta$-tubulin, and partial translation elongation factor 1- $\alpha$ (TEF) gene regions (Maharachchikumbura et al. 2014, 2016). Thus far, there are several species recorded in Pseudopestalotiopsis: Pseudopestalotiopsis camelliae, P. ignota, P. cocos, P. indica, P. kubahensis, $P$. ampullacea, $P$. chinensis, and $P$. theae (Lateef et al. 2015; Liu et al. 2017; Maharachchikumbura et al. 2014, 2016). Of these, $P$. theae

\footnotetext{
${ }^{\dagger}$ Corresponding authors: Y. Chen; E-mail: cyj8@ scu.edu.cn; and H. Tong; E-mail: thuarong@126.com
}

Accepted for publication 11 August 2017.

() 2018 The American Phytopathological Society and $P$. camelliae are considered to be economically significant species that have been reported in major tea-growing countries all over the world (Agnihothrudu 1964; Maharachchikumbura et al. 2016; Muraleedharan and Chen 1997). Neopestalotiopsis spp. having versicolorous median cells can be easily distinguished from Pseudopestalotiopsis spp. by gene sequence data and generally darkly colored concolorous median cells (Maharachchikumbura et al. 2014). Thus far, several Neopestalotiopsis isolates associated with $C$. sinensis have been reported by Liu et al. (2017). However, due to the unstable tree topologies, Neopestalotiopsis isolates were not classified (Liu et al. 2017). The objective of this study was to identify and characterize the causal agents of commonly observed gray blight symptoms on tea leaves and to compare the Pestalotiopsis-like species that have gone largely unrecognized.

\section{Materials and Methods}

Morphological analysis. More than 80 naturally infected tea leaves were collected from several commercial tea plantations located in the Chongqing district of China. Plant materials were surface disinfected, cut into small pieces $\left(4 \mathrm{~mm}^{2}\right)$, and placed in $9-\mathrm{cm}-$ diameters petri dishes containing $10 \mathrm{ml}$ of potato dextrose agar medium (PDA). All plates were incubated at $25 \pm 2{ }^{\circ} \mathrm{C}$ with $12 \mathrm{~h}$ of darkness. Cultural morphology was examined after 5 to 7 days. The morphological criteria of conidia such as size, shape, color and length of median cells, septation, and number of apical and basal appendages were recorded (Keith et al. 2006; Sutton 1980). Conidial size (length and width) and lengths of median cells and apical and basal appendages were determined by measuring arbitrarily selected conidia from a conidial suspension of each isolate that was prepared in sterile distilled water. Conidial characteristics of all isolates were observed with a U-TV0.5XC-3 microscope (Olympus) and 30 conidial measurements were taken for each isolate.

Polymerase chain reaction amplification and sequencing. The fungal genomic DNA was extracted from fresh mycelium by a cetyltrimethylammonium bromide method, according to the procedure of Than et al. (2008) and Chen et al. (2017a). The mycelium of each isolate was obtained from cultures grown on PDA for 5 days. Five pairs of primers were used for polymerase chain reaction (PCR) amplification (Table 1). The amplified products were separated on $1 \%$ agarose gel and purified using the Gel Extraction Kit (Omega Bio-Tek), according to the manufacturer's protocol. The purified PCR products were cloned into the pMD19-T Vector (TaKaRa). The positive transformants were sequenced at the Shanghai Sangon Biotech Company. 
Molecular characterization. To identify the isolates obtained in this study, sequences were subjected to BLAST search (https://blast.ncbi. nlm.nih.gov/Blast.cgi) with the National Center for Biotechnology Information (NCBI) database. Sixteen representative isolates (LB01, LB02, LB05-LB08, and LB11 to LB20) of this study were selected for phylogenetic analyses and their homologous ITS, $\beta$-tubulin, and TEF gene sequences of known species in the NCBI GenBank database were retrieved for comparison (Table 2). Phylogenetic trees were built and viewed in Molecular Evolutionary Genetic Analysis software, version 6.0, with both the maximum-parsimony (MP) and neighbor-joining (NJ) methods derived from individual and combined ITS, $\beta$-tubulin, and TEF gene sequences. Multiple sequence alignments of each gene were constructed using ClustalW and manually adjusted to allow maximum sequence similarity (Tamura et al. 2013). To determine the confidence values for the grouping within a tree, a bootstrap analysis was performed using 1,000 resamplings of the data (Saitou and Nei 1987; Tamura et al. 2013).

Pathogenicity tests. The pathogenicity of each isolate was tested on leaves of 2-year-old potted 'Fudingdabai' tea seedlings that were surfaced disinfected with $75 \%$ ethanol. For each isolate, 20 leaves on tea seedlings were inoculated. Conidial suspensions $(8 \mu \mathrm{l})$ were placed at wounded and nonwounded sites on the middle of the tea leaves (Chen et al. 2017a; Lin et al. 2002; Than et al. 2008). Wound inoculation involved pinpricking the middle of the leaves (two sites per leaf along the longitudinal axis) and then placing a conidial suspension over each wound. Conidial suspensions were prepared according to the procedure of Chen et al. (2017a). Tea leaves inoculated with only sterile water served as controls. Inoculated tea seedlings were covered with plastic bags to maintain high relative humidity for 2 days and maintained in the greenhouse at $25 \pm 2{ }^{\circ} \mathrm{C}$ until disease symptoms appeared. All fungal isolates included in pathogenicity tests were reisolated from the diseased tea leaves to confirm their identity with both molecular and morphological methods, as described above. Disease incidence (infected leaves) was assessed 7 to 15 days after inoculation by counting the number of diseased leaves compared with the total inoculated leaves. The virulence of the isolates was evaluated by measuring the diameter of the necrotic lesions which developed on tea leaves 7 days after wound inoculation. Experiments were repeated twice, with similar results. Statistical analysis was carried out with IBM SPSS Statistics 20.0 by one-way analysis of variance and means were compared by Tukey's honestly significant difference test $(P \leq 0.05)$.

\section{Results}

Disease survey. During the tea growing season of 2014 and 2015, gray blight was commonly observed on popular tea cultivars in tea plantations of Chongqing, China. Symptoms began as small brown spots that gradually expanded to large, circular to irregular black and brown necrotic lesions surrounded by a dark-brown border; in severe cases, lesions developed on large portions of a single leaf and, eventually, the dead leaves fell from the tea plants (Fig. 1A). Dark acervuli formed on the upper surface of the lesions and the color of necrotic lesions changed to light gray at later stages of disease.

Cultural characteristics. In total, 16 isolates were selected from more than 80 isolates for further identification based on differences in field symptoms and cultural morphology on PDA. The isolates were placed into three groups based on colony characteristics. Among these, eight isolates (LB01, LB02, LB05 to LB08, LB15, and LB16), producing conidia with brown or olivaceous concolorous median cells, were identified as the species of Pseudopestalotiopsis (Fig. 2A); four isolates (LB11 to LB14), producing conidia with versicolorous median cells, belonged to the genus Neopestalotiopsis (Fig. 2B); and the remaining four isolates (LB17 to LB20), producing conidia with light to dark-brown concolorous median cells but without basal appendages, were identified as Pestalotiopsis (Fig. 2C). All of the isolates of Pseudopestalotiopsis and Neopestalotiopsis cultured on PDA grew quickly, attaining 76 to $85 \mathrm{~mm}$ in diameter after 5 days at $25^{\circ} \mathrm{C}$. Colonies of these two genera were white to creamy; composed of concentric rings; with undulate edge, whitish, dense aerial mycelium on the surface; and white to pale orange color diffused in the reverse side (Fig. 1B and C). By contrast, the colonies of Pestalotiopsis (LB17 to LB20) on PDA had no concentric rings, a smooth edge, and thin mycelium (Fig. 1D). The conidiomata of all isolates formed on the surface were black, gregarious, and diffused throughout the culture mediums (Fig. 1B, C, and D).

Conidial morphology. All of the isolates belonging to the genera Pseudopestalotiopsis and Neopestalotiopsis produced five-celled conidia that were fusiform, straight or slightly curved, with threecolored median cells and two colorless terminal cells as well as two to four hyaline apical appendages (most were three, rarely two or four) and one hyaline basal appendage arising from the apex (Fig. 2A and B). Lengths of basal appendages of the isolates in two groups were similar. However, the isolates in two genera can be clearly and easily differentiated by the size of conidia, the length of apical appendages, and the color and length of median cells. The conidial size of Pseudopestalotiopsis isolates ranged from 22.5 to $36.0 \mu \mathrm{m}$ in length by 4.3 to $8.5 \mu \mathrm{m}$ in width, and were much longer than those of Neopestalotiopsis isolates (Table 3). Lengths of apical appendages of Pseudopestalotiopsis were 15.1 to $38.0 \mu \mathrm{m}$, distinctly longer than those of the isolates in genus Neopestalotiopsis, which were 12.2 to $23.4 \mu \mathrm{m}$. The three median cells of isolates in genus Pseudopestalotiopsis were concolorous, dark brown or olivaceous (Fig. 2A), with the length ranging from 15.6 to $26.0 \mu \mathrm{m}$ (Table 3), whereas the median cells of Neopestalotiopsis were versicolorous, the upper two cells were dark brown, and the lowest one was pale brown (Fig. 2B), with the length ranging from 11.0 to $17.2 \mu \mathrm{m}$ (Table 3). In contrast, although the isolates of Pestalotiopsis also produced concolorous median cells, the color of median cells was light to dark brown, the basal appendages arising from the apex were absent, and the number of hyaline apical appendages was three to seven (Fig. 2C), all of which distinguished this genus from the other two.

Phylogenetic analysis. Five gene regions of ITS, $\beta$-tubulin, TEF, glyceraldehyde-3-phosphate dehydrogenase, and actin were utilized to resolve species delimitation but, due to the failure in PCR amplification or in their ability to resolve species boundaries, only ITS, $\beta$-tubulin, and TEF proved to be useful markers for phylogenetic analysis. Sequences obtained in this study were compared with homologous gene sequences retrieved from the NCBI GenBank database (Table 2). Phylogenetic trees were performed by both MP and

Table 1. Primer pairs used to amplify gene regions of Pestalotiopsis-like species

\begin{tabular}{|c|c|c|c|}
\hline Gene $^{\mathbf{z}}$ & Primer & Gene sequence & Reference \\
\hline \multirow{2}{*}{ ITS } & ITS4 & 5'-TCCTCCGCTTATTGATATGC-3' & White et al. 1990 \\
\hline & ITS5 & 5'-GGAAGTAAAAGTCGTAACAAGG-3' & $\ldots$ \\
\hline \multirow[t]{2}{*}{$\beta$-tubulin } & $\mathrm{T} 1$ & 5'-AACATGCGTGAGATTGTAAGT-3' & O’Donnell and Cigelnik 1997 \\
\hline & $\beta \mathrm{t} 2 \mathrm{~b}$ & 5'-ACCCTCAGTGTAGTGACCCTTGGC-3' & \\
\hline \multirow[t]{2}{*}{ TEF } & EF1-526F & 5'-GTCGTYGTYATYGGHCAYGT-3' & Rehner 2001 \\
\hline & EF1-1567R & 5'-ACHGTRCCRATACCACCRATCTT-3' & $\ldots$ \\
\hline \multirow[t]{2}{*}{ GAPDH } & GDF1 & 5'-GCCGTCAACGACCCCTTCATTGA-3' & Guerber et al. 2003 \\
\hline & GDR1 & 5'-GGGTGGAGTCGTACTTGAGCATGT-3' & $\ldots$ \\
\hline \multirow[t]{2}{*}{ ACT } & ACT-512F & 5'-ATGTGCAAGGCCGGTTTCGC-3' & Carbone and Kohn 1999 \\
\hline & ACT-783R & 5'-TACGAGTCCTTCTGGCCCAT-3' & $\ldots$ \\
\hline
\end{tabular}

${ }^{\mathrm{z}} \mathrm{ITS}=$ internal transcribed spacer, TEF $=$ translation elongation factor $1-\alpha, \mathrm{GAPDH}=$ glyceraldehyde-3-phosphate dehydrogenase, and ACT $=$ actin. 
Table 2. Species isolated from tea plants and the reference isolates used for sequence comparisons and phylogenetic analyses

\begin{tabular}{|c|c|c|c|c|c|}
\hline \multirow[b]{2}{*}{ Species, isolates } & \multirow[b]{2}{*}{ Host plant } & \multirow[b]{2}{*}{ Geographical origin } & \multicolumn{3}{|c|}{ GenBank accession number ${ }^{\mathrm{z}}$} \\
\hline & & & ITS & $\beta$-Tubulin & TEF \\
\hline \multicolumn{6}{|c|}{ Pseudopestalotiopsis camelliae-sinensis } \\
\hline LB01 & Camellia sinensis & China & KX757707 & KX757720 & KY342347 \\
\hline LB02 & C. sinensis & China & KX757708 & KX757721 & KY342348 \\
\hline LB05 & C. sinensis & China & KX757711 & KX757724 & KY342351 \\
\hline LB06 & C. sinensis & China & KX757712 & KX757725 & KY342352 \\
\hline LB07 & C. sinensis & China & KX757713 & KX757726 & KY342353 \\
\hline LB08 & C. sinensis & China & KX757714 & KX757727 & KY342354 \\
\hline LB15 & C. sinensis & China & KY319132 & KY363537 & KY342357 \\
\hline LB16 & C. sinensis & China & KY319133 & KY363538 & KY342358 \\
\hline LC3571 & C. sinensis & China & KX894986 & KX895317 & KX895203 \\
\hline LC3023 & C. sinensis & China & KX894943 & KX895275 & KX895160 \\
\hline LC3022 & C. sinensis & China & KX894942 & KX895274 & KX895159 \\
\hline LC3020 & C. sinensis & China & KX894940 & KX895272 & KX895157 \\
\hline LC3009 & C. sinensis & China & KX894935 & KX895267 & KX895152 \\
\hline LC3010 & C. sinensis & China & KX894936 & KX895268 & KX895153 \\
\hline \multicolumn{6}{|l|}{ P. camelliae } \\
\hline CGMCC 3.9192 & C. sinensis & China & JN943622 & KU562851 & KU562850 \\
\hline \multicolumn{6}{|l|}{ P. theae } \\
\hline MAFF 752011 & Thea sinensis & Japan: Tokushima & AB482210 & AB453902 & AB453858 \\
\hline \multicolumn{6}{|l|}{ P. chinensis } \\
\hline LC6695 & C. sinensis & China & KX895031 & KX895364 & KX895249 \\
\hline LC6711 & C. sinensis & China & KX895032 & KX895365 & KX895250 \\
\hline LC6629 & C. sinensis & China & KX895026 & KX895359 & KX895245 \\
\hline \multicolumn{6}{|l|}{ P. smitheae } \\
\hline MFLUCC 12-0121 & Pandanus odoratissimus & Thailand & KJ503812 & KJ503815 & KJ503818 \\
\hline MFLUCC 12-0125 & Living leaves of Pandanus & Thailand & KJ503813 & KJ503816 & KJ503819 \\
\hline \multicolumn{6}{|l|}{$P . \operatorname{cocos}$} \\
\hline CBS 272.29 & Cocos nucifera & Indonesia & KM199378 & KM199467 & KM199553 \\
\hline \multicolumn{6}{|l|}{ P. ampullaceal } \\
\hline LC6618 & Camellia sinensis & China & KX895025 & KX895358 & KX895244 \\
\hline \multicolumn{6}{|l|}{ P. indica } \\
\hline CBS 459.78 & Hibiscus rosa-sinensis & India & KM199381 & KM199470 & KM199560 \\
\hline \multicolumn{6}{|c|}{ Pseudopestalotiopsis sp. 3-KW-2016 } \\
\hline 14 & Unknown & Myanmar & LC114034 & LC114054 & LC114074 \\
\hline Pseudopestalotiopsis s & & & & & \\
\hline $\mathrm{SC} 5 \mathrm{~A} 7$ & C. sinensis & China & KX146657 & KX146775 & KX146716 \\
\hline HUN1A5 & C. sinensis & China & KX146646 & KX146764 & KX146705 \\
\hline GX1D13 & C. sinensis & China & KX146645 & KX146763 & KX146704 \\
\hline Neopestalotiopsis clav & & & & & \\
\hline LB11 & C. sinensis & China & KX757717 & KX757730 & KY342359 \\
\hline LB12 & C. sinensis & China & KX757718 & KX757731 & KY342360 \\
\hline LB13 & C. sinensis & China & KX757719 & KX757732 & KY342361 \\
\hline LB14 & C. sinensis & China & KY319134 & KY363536 & KY342362 \\
\hline CBS 447.73 & Decaying wood & Sri Lanka & KM199374 & KM199443 & KM199539 \\
\hline N. samarangensis & & & & & \\
\hline CBS 115451 & Unidentified tree & Hong Kong & KM199365 & KM199447 & KM199556 \\
\hline N. piceana & & & & & \\
\hline CBS 225.30 & Mangifera indica & - & KM199371 & KM199451 & KM199535 \\
\hline CBS 254.32 & Cocos nucifera & Indonesia: Sulawesi & KM199372 & KM199452 & KM199529 \\
\hline CBS 394.48 & Picea sp. & United Kingdom & KM199368 & KM199453 & KM199527 \\
\hline N. rosae & & & & & \\
\hline CBS 101057 & Rosa sp. & New Zealand & KM199359 & KM199429 & KM199523 \\
\hline CBS 124745 & Paeonia suffruticosa & United States & KM199360 & KM199430 & KM199524 \\
\hline N. ellipsospora & & & & & \\
\hline CBS 115113 & Ardisia crenata & Hong Kong & KM199343 & KM199450 & KM199544 \\
\hline N. javaensis & & & & & \\
\hline CBS 257.31 & C. nucifera & Indonesia: Java & KM199357 & KM199437 & KM199543 \\
\hline N. mesopotamica & & & & & \\
\hline CBS 336.86 & Pinus brutia & Iraq & KM199362 & KM199441 & KM199555 \\
\hline CBS 299.74 & Eucalyptus sp. & Turkey & KM199361 & KM199435 & KM199541 \\
\hline Neopestalotiopsis sp. & & & & & \\
\hline CBS 664.94 & C. nucifera & The Netherlands & KM199354 & KM199449 & KM199525 \\
\hline CBS 177.25 & Dalbergia sp. & - & KM199370 & KM199445 & KM199533 \\
\hline CBS 274.29 & C. nucifera & Indonesia: Java & KM199375 & KM199448 & KM199534 \\
\hline & & & & (contine & n next page) \\
\hline
\end{tabular}

\footnotetext{
${ }^{\mathrm{z}}$ ITS = internal transcribed spacer and TEF = translation elongation factor $1-\alpha$.
} 
Table 2. (continued from previous page)

\begin{tabular}{|c|c|c|c|c|c|}
\hline \multirow[b]{2}{*}{ Species, isolates } & \multirow[b]{2}{*}{ Host plant } & \multirow[b]{2}{*}{ Geographical origin } & \multicolumn{3}{|c|}{ GenBank accession number ${ }^{z}$} \\
\hline & & & ITS & $\beta$-Tubulin & TEF \\
\hline LC6320 & Camellia sinensis & China & KX895018 & KX895351 & KX895237 \\
\hline LC3318 & C. sinensis & China & KX894964 & KX895296 & KX895181 \\
\hline LC6489 & C. sinensis & China & KX895020 & KX895353 & KX895239 \\
\hline LC6288 & C. sinensis & China & KX895014 & KX895347 & KX895233 \\
\hline \multicolumn{6}{|l|}{ N. formicarum } \\
\hline CBS 115.83 & Plant debris & Cuba & KM199344 & KM199444 & KM199519 \\
\hline CBS 362.72 & Dead Formicidae (ant) & Ghana & KM199358 & KM199455 & KM199517 \\
\hline \multicolumn{6}{|l|}{ N. aotearoa } \\
\hline CBS 367.54; ATCC 11763; QM 381 & Canvas & New Zealand & KM199369 & KM199454 & KM199526 \\
\hline \multicolumn{6}{|l|}{ Pestalotiopsis camelliae } \\
\hline LB17 & C. sinensis & China & KY319135 & KY363539 & KY342363 \\
\hline LB18 & C. sinensis & China & KY319136 & KY363540 & KY342364 \\
\hline LB19 & C. sinensis & China & KY319137 & KY363541 & KY342365 \\
\hline LB20 & C. sinensis & China & KY319138 & KY363542 & KY342366 \\
\hline LC3154 & C. sinensis & China & KX895176 & KX895291 & KX895176 \\
\hline LC3346 & C. sinensis & China & KX894970 & KX895302 & KX895187 \\
\hline LC3343 & C. sinensis & China & KX894969 & KX895301 & KX895186 \\
\hline LC3348 & C. sinensis & China & KX894971 & KX895303 & KX895188 \\
\hline CBS 443.62 & C. sinensis & Turkey & KM199336 & KM199424 & KM199512 \\
\hline \multicolumn{6}{|l|}{ P. yanglingensis } \\
\hline LC3412 & C. sinensis & China & KX894980 & KX895312 & KX895197 \\
\hline LC3153 & C. sinensis & China & KX894958 & KX895290 & KX895175 \\
\hline LC3152 & C. sinensis & China & KX894957 & KX895289 & KX895174 \\
\hline \multicolumn{6}{|l|}{ Pestalotiopsis sp. } \\
\hline CBS 263.33 & Rhododendron ponticum & The Netherlands & KM199316 & KM199414 & KM199489 \\
\hline CBS 264.33 & Cocos sp. & Indonesia: Sulawesi & KM199322 & KM199412 & KM199490 \\
\hline \multicolumn{6}{|l|}{ P. papuana } \\
\hline CBS 331.96 & Coastal soil & Papua New Guinea & KM199321 & KM199413 & KM199491 \\
\hline CBS 887.96 & Cocos sp. & Papua New Guinea & KM199318 & KM199415 & KM199492 \\
\hline \multicolumn{6}{|l|}{ P. diploclisia } \\
\hline CBS 115449 & Psychotria & Hong Kong & KM199314 & KM199416 & KM199485 \\
\hline CBS 115585 & Diploclisia & Hong Kong & KM199315 & KM199417 & KM199483 \\
\hline CBS 115587 & Diploclisia & Hong Kong & KM199320 & KM199419 & KM199486 \\
\hline \multicolumn{6}{|l|}{ P. malayana } \\
\hline CBS 102220 & Macaranga & Malaysia & KM199306 & KM199411 & KM199482 \\
\hline \multicolumn{6}{|l|}{ P. monochaeta } \\
\hline CBS 144.97 & Quercus robur & The Netherlands & KM199327 & KM199386 & KM199479 \\
\hline CBS 440.83 & Taxus baccata & The Netherlands & KM199329 & KM199387 & KM199480 \\
\hline P. hollandica & & & & & \\
\hline CBS 265.33 & Sciadopitys verticillata & The Netherlands & KM199328 & KM199388 & KM199481 \\
\hline P. furcata & & & & & \\
\hline LC6601 & C. sinensis & China & KX895024 & KX895357 & KX895243 \\
\hline P. grevilleae & & & & & \\
\hline CBS 114127; STE-U 2919 & Grevillea sp. & Australia & KM199300 & KM199407 & KM199504 \\
\hline P. novae-hollandiae & & & & & \\
\hline CBS 130973 & Banksia grandis & Australia & KM199337 & KM199425 & KM199511 \\
\hline P. oryzae & & & & & \\
\hline CBS 353.69 & Oryza sativa & Denmark & KM199299 & KM199398 & KM199496 \\
\hline CBS 171.26 & Unknown & Italy & KM199304 & KM199397 & KM199494 \\
\hline CBS 111522; STE-U 2083 & Telopea sp. & Hawaii & KM199294 & KM199394 & KM199493 \\
\hline P. telopeae & & & & & \\
\hline CBS 113606; STE-U 3082 & Telopea sp. & Australia & KM199295 & KM199402 & KM199498 \\
\hline CBS 114161; STE-U 3083 & Telopea sp. & Australia & KM199296 & KM199403 & KM199500 \\
\hline P. australasiae & & & & & \\
\hline CBS 114141; STE-U 2949 & Protea sp. & Australia & KM199298 & KM199410 & KM199501 \\
\hline P. biciliata & & & & & \\
\hline CBS 790.68 & Taxus & The Netherlands & KM199305 & KM199400 & KM199507 \\
\hline CBS 124463 & Platanus & Slovakia & KM199308 & KM199399 & KM199505 \\
\hline CBS 236.38 & Paeonia & Italy & KM199309 & KM199401 & KM199506 \\
\hline P. knightiae & & & & & \\
\hline CBS 111963; STE-U 2905 & Knightia & New Zealand & KM199311 & KM199406 & KM199495 \\
\hline P. kenyana & & & & & \\
\hline CBS 442.67 & Coffea sp. & Kenya & KM199302 & KM199395 & KM199502 \\
\hline CBS 911.96 & Raw material from agar-agar & - & KM199303 & KM199396 & KM199503 \\
\hline
\end{tabular}




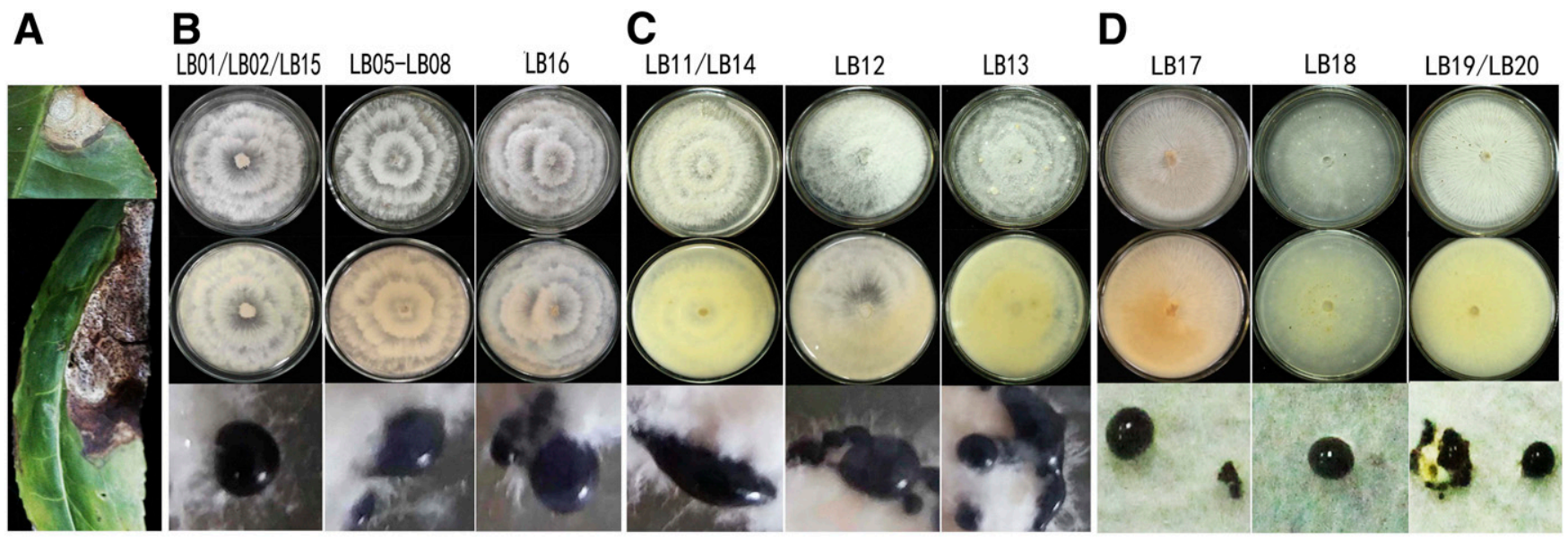

Fig. 1. Symptoms of gray blight on naturally infected tea leaves and the cultural traits of species isolated from symptomatic leaves. A, Typical symptoms caused by Pestalotiopsislike species and cultural traits of B, Pseudopestalotiopsis; B, Neopestalotiopsis; and D, Pestalotiopsis isolates on potato dextrose agar at $25^{\circ} \mathrm{C}$ for 7 days.

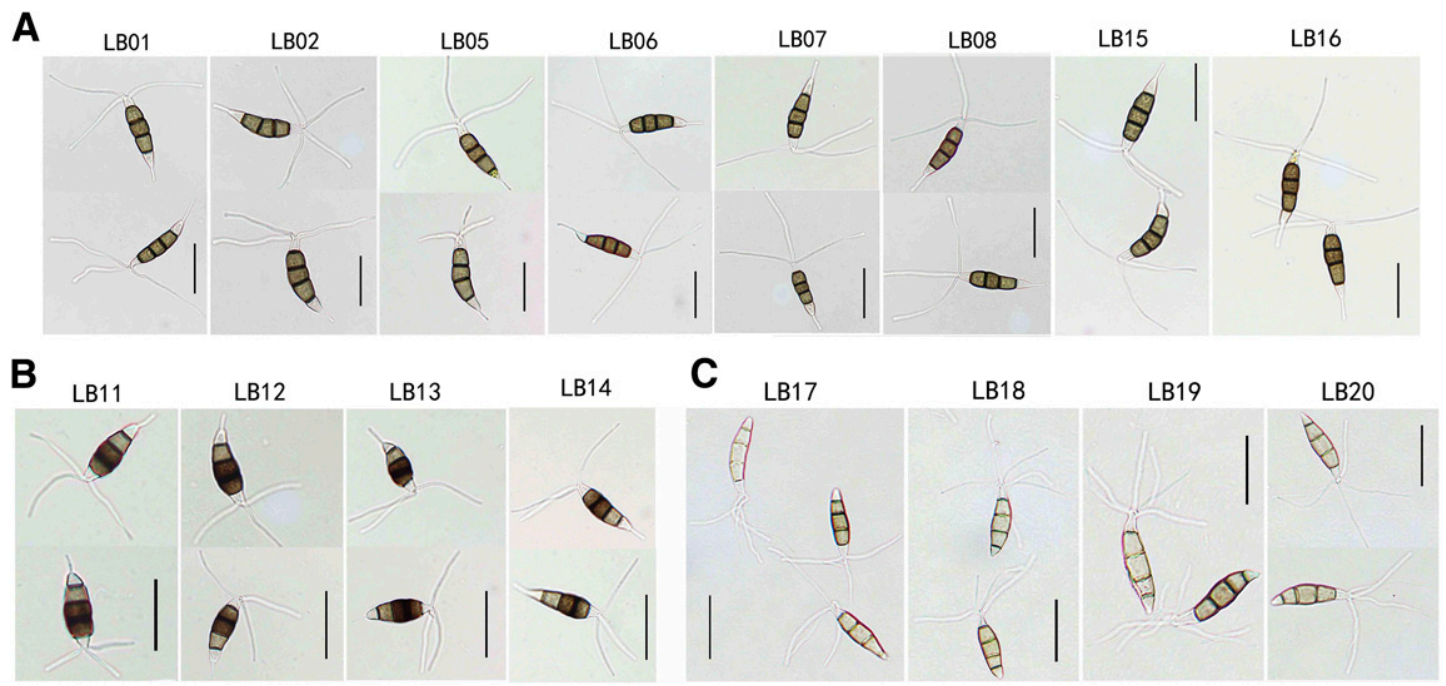

Fig. 2. Conidial morphology of A, 12 isolates of Pseudopestalotiopsis; B, 4 isolates of Neopestalotiopsis; and C, 4 isolates of Pestalotiopsis on potato dextrose agar at $25^{\circ} \mathrm{C}$ for 10 days (scale bar $=20 \mu \mathrm{m}$ ).

Table 3. Conidial characteristics of the Pestalotiopsis-like species in the present study ${ }^{\mathrm{y}}$

\begin{tabular}{|c|c|c|c|c|c|c|c|}
\hline Species, isolate & CL $(\mu \mathrm{m})$ & $\mathbf{C W}(\mu \mathbf{m})$ & Median cells $\mathbf{z}$ & Middle ( $\mu \mathrm{m})$ & Apical $(\mu \mathrm{m})$ & Basal $(\mu \mathbf{m})$ & $N$ \\
\hline \multicolumn{8}{|c|}{ Pseudopestalotiopsis camelliae-sinensis } \\
\hline LB01 & $22.5-34.0$ & $5.2-7.8$ & Con, DB, O & $16.5-22.0$ & $25.1-38.0$ & $4.2-9.3$ & $3-4$ \\
\hline LB02 & $24.2-35.0$ & $6.2-8.5$ & Con, DB, O & $17.8-24.1$ & $22.0-36.1$ & $4.8-7.8$ & $3-4$ \\
\hline LB05 & $27.3-36.0$ & $5.4-8.4$ & Con, $\mathrm{DB}, \mathrm{O}$ & $16.8-24.1$ & $16.2-36.1$ & $4.8-8.1$ & $3-4$ \\
\hline LB06 & $25.6-32.0$ & $5.1-7.8$ & Con, $\mathrm{DB}, \mathrm{O}$ & $17.1-22.1$ & $25.1-35.2$ & $5.8-8.3$ & $3-4$ \\
\hline LB07 & $23.2-31.0$ & $4.3-7.2$ & Con, $\mathrm{DB}, \mathrm{O}$ & $17.2-24.1$ & $15.1-38.0$ & $5.6-8.9$ & $3-4$ \\
\hline LB08 & $28.1-33.0$ & $5.7-7.9$ & Con, $\mathrm{DB}, \mathrm{O}$ & $16.8-24.0$ & $26.0-31.1$ & $5.2-8.2$ & $2-4$ \\
\hline LB15 & $26.7-34.0$ & $5.4-7.4$ & Con, $\mathrm{DB}, \mathrm{O}$ & $17.2-26.0$ & $25.0-35.0$ & $4.3-7.9$ & $3-4$ \\
\hline LB16 & $24.7-33.1$ & $5.1-7.7$ & Con, $\mathrm{DB}, \mathrm{O}$ & $15.6-23.1$ & $26.1-37.0$ & $5.1-7.8$ & 3 \\
\hline \multicolumn{8}{|c|}{ Neopestalotiopsis clavispora } \\
\hline LB11 & $19.8-22.6$ & $6.1-8.9$ & Ver B, PB & $13.5-16.7$ & $12.2-22.5$ & $5.3-8.5$ & $3-4$ \\
\hline LB12 & $18.1-23.6$ & $5.9-8.3$ & Ver B, PB & $12.6-17.2$ & $16.4-19.6$ & $4.8-7.2$ & $3-4$ \\
\hline LB13 & $16.3-22.0$ & $5.8-8.8$ & Ver B, PB & $11.0-15.8$ & $15.5-22.7$ & $5.0-8.2$ & 3 \\
\hline LB14 & $16.3-23.7$ & $5.5-8.4$ & Ver B, PB & $13.7-17.1$ & $15.8-23.4$ & $4.8-7.4$ & $3-4$ \\
\hline \multicolumn{8}{|c|}{ Pestalotiopsis camelliae } \\
\hline LB17 & $22.1-30.0$ & $3.6-6.2$ & Con, L, DB & $13.5-18.2$ & $14.6-29.1$ & $\ldots$ & 3-7 \\
\hline LB18 & $19.2-31.0$ & $3.3-6.4$ & Con, L, DB & $12.5-18.9$ & $13.5-28.9$ & $\ldots$ & $3-7$ \\
\hline LB19 & $23.6-33.0$ & $4.3-6.8$ & Con, L, DB & $14.9-22.3$ & $14.3-32.4$ & $\ldots$ & $3-7$ \\
\hline LB20 & $21.1-32.0$ & $3.5-6.7$ & Con, L, DB & $14.5-21.9$ & $15.2-31.8$ & $\ldots$ & 3-7 \\
\hline
\end{tabular}

${ }^{\mathrm{y}}$ Characteristics: $\mathrm{CL}=$ conidia length, $\mathrm{CW}=$ conidia width, Middle $=$ length of middle cells, Apical = apical appendage length, Basal = basal appendage length, and $N=$ number of apical appendages.

${ }^{\mathrm{z}}$ Abbreviations: Con, DB, O = concolorous, dark brown, or olivaceous; Ver, B, PB = versicolorous, the upper two brown, the lowest pale brown; and Con, $\mathrm{L}$, $\mathrm{DB}=$ concolorous, light to dark brown. 


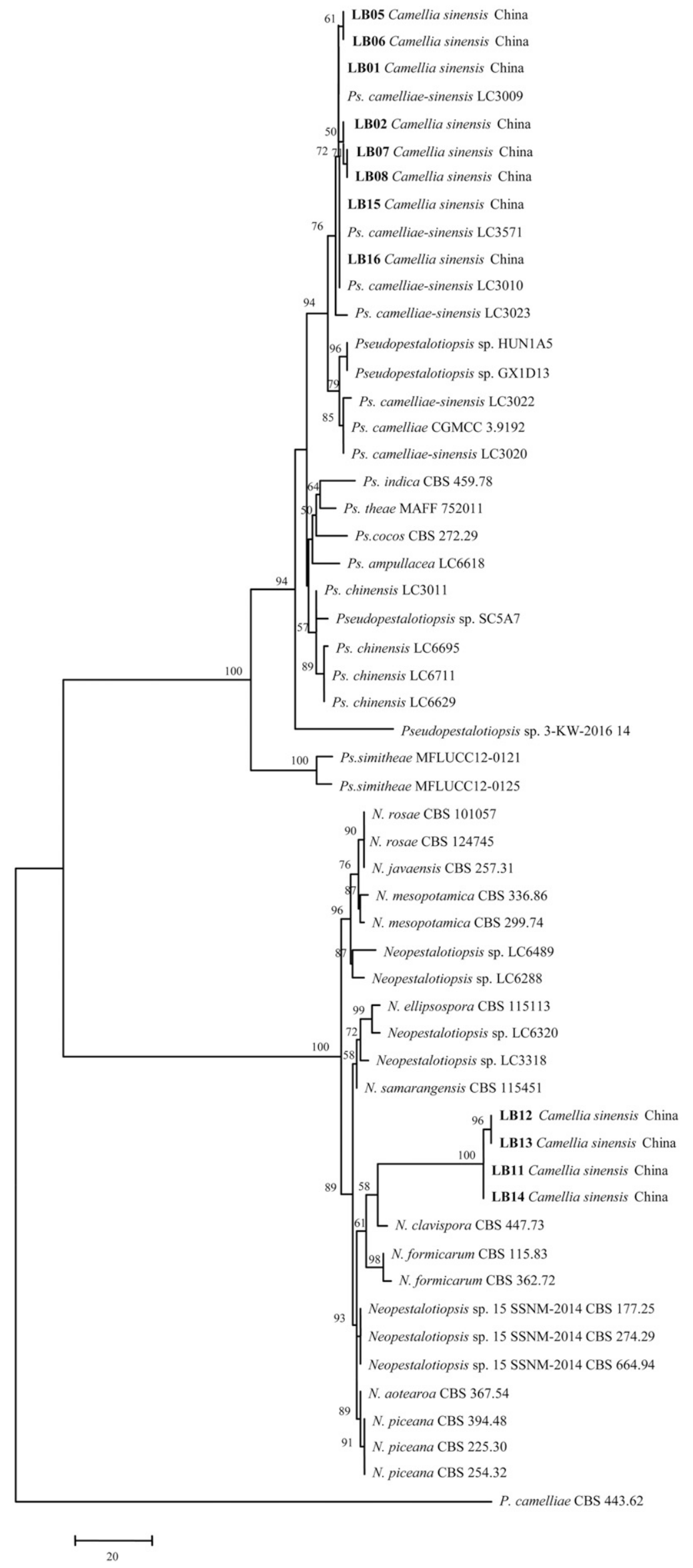

Fig. 3. Molecular phylogenetic tree of Pseudopestalotiopsis and Neopestalotiopsis spp. obtained through maximum-parsimony method based on the combined internal transcribed spacer, $\beta$-tubulin, and translation elongation factor $1-\alpha$ gene sequences from collected isolates in the present study (bold font) and published sequences. Pseudopestalotiopsis camelliae CBS 443.62 was used as the outgroup. Numbers in the bootstrap test (1,000 replicates) are shown above the branches and only values above $50 \%$ are shown. 


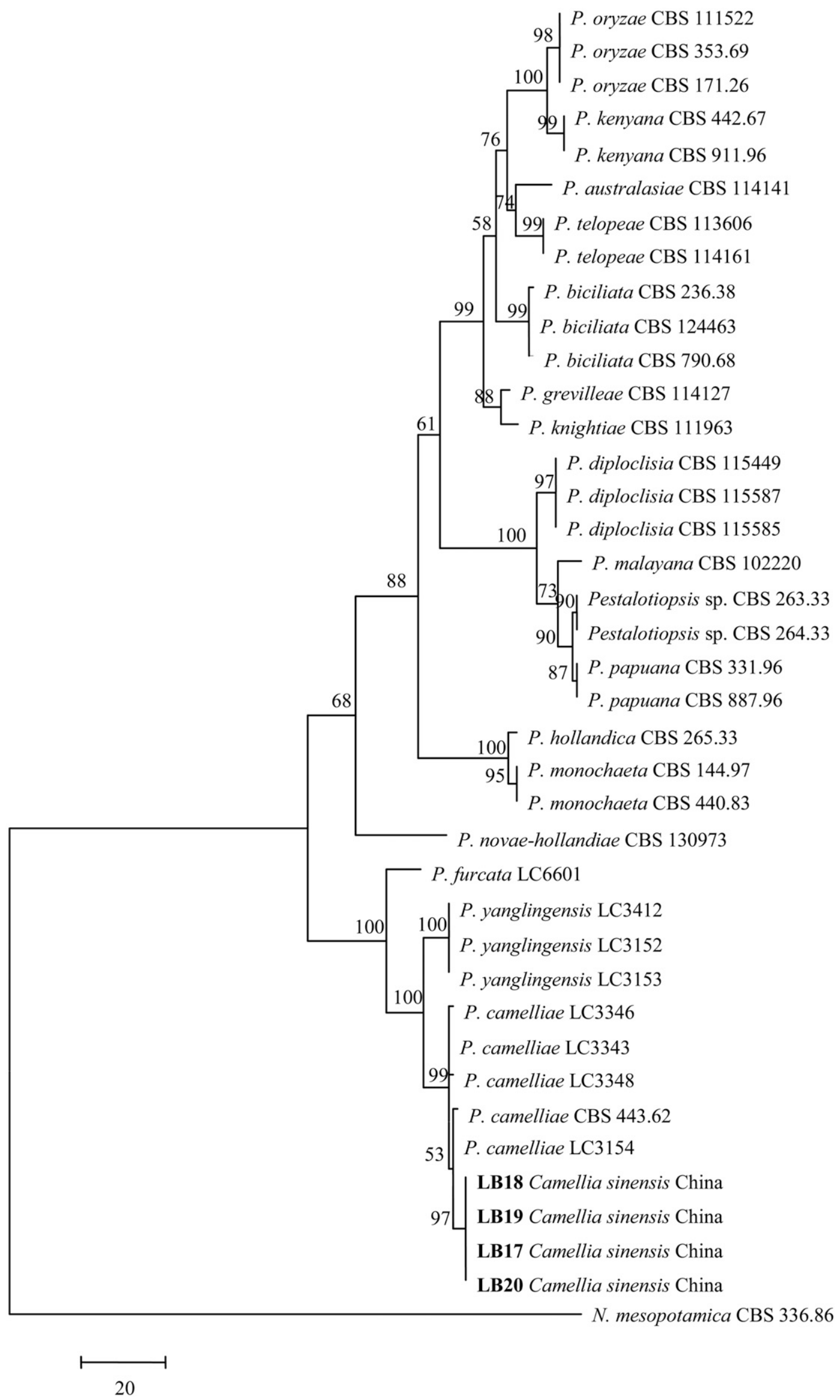

Fig. 4. Molecular phylogenetic tree of Pestalotiopsis spp. obtained through maximum-parsimony method based on the combined ITS, $\beta$-tubulin, and translation elongation factor 1 $\alpha$ gene sequences from collected isolates in the present study (bold font) and published sequences. Neopestalotiopsis mesopotamica CBS 336.86 was used as the outgroup. Numbers in the bootstrap test (1,000 replicates) are shown above the branches and only values above $50 \%$ are shown 
$\mathrm{NJ}$ methods using individual and combined ITS, $\beta$-tubulin, and TEF gene sequences. However, some species could not be differentiated from closely related species using any single gene sequence dataset (data not shown). Therefore, the concatenated dataset of ITS, $\beta$-tubulin, and TEF were used to resolve all the isolates analyzed in this study. Phylogenetic trees performed by both MP and NJ methods produced similar topologies; thus, only trees constructed by MP method were shown here (Figs. 3 and 4).

The MP tree derived from 54 isolates of Pseudopestalotiopsis and Neopestalotiopsis indicated that all of the isolates were distinctly separated into two main clusters with high bootstrap supports (Fig. 3). Eight Pseudopestalotiopsis isolates in this study (LB01, LB02, LB05 to LB08, LB15, and LB16) were grouped into one cluster containing $P$. camelliae-sinensis, $P$. chinensis, $P$. theae, $P$. cocos, $P$. ampullacea, and $P$. indica, but all isolates were closely related to a new species, $P$. camelliae-sinensis (Liu et al. 2017) obtained from C. sinensis; whereas isolates LB11 to LB14 grouped with the other cluster comprising several Neopestalotiopsis spp., with a high bootstrap value $100 \%$, and were more related to Neopestalotiopsis clavispora. Based on phylogenetic analysis and morphological characteristics, the isolates of Pseudopestalotiopsis known to possess concolorous median cells and Neopestalotiopsis isolates known to possess versicolorous median cells were described as $P$. camelliae-sinensis and $N$. clavispora, respectively.

The MP tree constructed by the combined ITS, $\beta$-tubulin, and TEF genes of 38 Pestalotiopsis spp. indicated that the four Pestalotiopsis isolates in this study (LB17 to LB20) were grouped together with Pestalotiopsis camelliae obtained from tea leaves of $C$. sinensis in China (Liu et al. 2017) and Turkey (Maharachchikumbura et al. 2014), with a high bootstrap value 99\% (Fig. 4). Combined with morphological characteristics, the isolates of Pestalotiopsis known to possess concolorous median cells but having no basal appendage were identified as $P$. camelliae.

The pathogenicity of Pestalotiopsis-like species. Results of pathogenicity tests showed that, with wound inoculation, all isolates in this study were pathogenic on tea leaves (Table 4) and the symptoms induced were similar to those which occur under natural conditions in the field. The percentage of infected tea leaves on wounded inoculated tea leaves for the 16 isolates all ranged from 41.7 to $76.7 \%$ by 15 days after inoculation (Table 4). In contrast, symptoms were not usually observed on nonwounded leaves (Table 4), suggesting that wounding may be necessary for symptom development. All of the representative isolates of Pseudopestalotiopsis camelliae-sinensis and $N$. clavispora were highly pathogenic to the artificially inoculated tea leaves and showed no significant difference in lesion size between the two species, whereas the isolates of Pestalotiopsis camelliae were less virulent (Table 4). The fungi were reisolated from lesions of the diseased leaves with $100 \%$ frequency, and their morphological characteristics and gene sequences were identical to the original ones, which confirmed that all three species (Pseudopestalotiopsis camelliae-sinensis, N. clavispora, and Pestalotiopsis camelliae) are the causal agents for tea gray blight disease.

\section{Discussion}

Gray leaf blight of tea is an economically important disease caused by Pestalotiopsis-like fungi. At this time, there is limited information about the identity of these pathogens in the major tea-growing countries of the world. Pestalotiopsis spp., as potent plant pathogens, have gone largely unrecognized using only morphological or molecular data (Hu et al. 2007; Maharachchikumbura et al. 2011, 2012, 2013a,b), because colony morphology such as color, growth rate, and texture tends to be highly variable within species (Jeewon et al. 2003; Keith et al. 2006) and most DNA sequences deposited for Pestalotiopsis spp. in GenBank are likely to have been wrongly named or confused (Maharachchikumbura et al. 2011). Furthermore, $\mathrm{Hu}$ et al. (2007) showed that the ITS region is less informative than the $\beta$-tubulin gene in differentiating Pestalotiopsis spp. but Liu et al. (2010) disagreed and indicated that proper analysis and alignment of the ITS region can group Pestalotiopsis spp. according to different types of conidia. In 2014, a revision of Pestalotiopsis sensu lato was done by Maharachchikumbura et al. (2014) in "Pestalotiopsis revisited", in which they segregated two novel genera from Pestalotiopsis (namely, Neopestalotiopsis and Pseudopestalotiopsis) based on the combined morphological and DNA data. In the present study, although all of the isolates from tea plants could be distinctly separated into three genera by morphological characteristics or any individual ITS, $\beta$-tubulin, and TEF sequence data, some species could not be differentiated from closely related species because fewer homologous gene sequences were available for sequence comparison or some of the sequences in this study contained low similarity to other sequences in the NCBI GenBank database. Therefore, combined multigene data of ITS, $\beta$-tubulin, and TEF and distinct morphological characteristics may

Table 4. Pathogenicity of 16 representative isolates from tea gray blight

\begin{tabular}{|c|c|c|c|}
\hline \multirow[b]{2}{*}{ Species, isolate } & \multicolumn{2}{|c|}{ Infected leaves $(\%)^{\mathrm{y}}$} & \multirow{2}{*}{$\frac{\text { Lesion diameter }(\mathrm{mm})^{\mathrm{z}}}{\text { Wounded }}$} \\
\hline & Wounded & Nonwounded & \\
\hline \multicolumn{4}{|c|}{ Pseudopestalotiopsis camelliae-sinensis } \\
\hline LB01 & $60.0 \pm 10.0$ & NS & $9.2 \pm 0.9 \mathrm{a}$ \\
\hline LB02 & $63.0 \pm 7.6$ & NS & $9.6 \pm 1.1 \mathrm{a}$ \\
\hline LB05 & $66.7 \pm 12.6$ & NS & $9.9 \pm 0.9 \mathrm{a}$ \\
\hline LB06 & $61.7 \pm 12.6$ & $3.3 \pm 5.8$ & $10.5 \pm 1.3 \mathrm{a}$ \\
\hline LB07 & $73.3 \pm 2.9$ & NS & $10.1 \pm 0.9 \mathrm{a}$ \\
\hline LB08 & $76.7 \pm 10.4$ & $1.67 \pm 2.9$ & $10.8 \pm 1.3 \mathrm{a}$ \\
\hline LB15 & $50.0 \pm 10.0$ & NS & $10.1 \pm 0.9 \mathrm{a}$ \\
\hline LB16 & $41.7 \pm 10.4$ & NS & $8.7 \pm 0.8 \mathrm{a}$ \\
\hline \multicolumn{4}{|c|}{ Neopestalotiopsis clavispora } \\
\hline LB11 & $58.3 \pm 7.6$ & NS & $9.7 \pm 1.0 \mathrm{a}$ \\
\hline LB12 & $75.0 \pm 5.0$ & NS & $10.5 \pm 1.6 \mathrm{a}$ \\
\hline LB13 & $70.0 \pm 10.0$ & NS & $10.7 \pm 0.9 \mathrm{a}$ \\
\hline LB14 & $55.0 \pm 8.7$ & NS & $8.9 \pm 1.0 \mathrm{a}$ \\
\hline \multicolumn{4}{|c|}{ Pestalotiopsis camelliae } \\
\hline LB17 & $46.7 \pm 10.4$ & NS & $4.7 \pm 0.5 b$ \\
\hline LB18 & $51.7 \pm 7.6$ & NS & $3.3 \pm 0.7 \mathrm{~b}$ \\
\hline LB19 & $48.3 \pm 7.6$ & NS & $5.3 \pm 0.9 b$ \\
\hline LB20 & $60.0 \pm 10.0$ & NS & $4.9 \pm 1.0 \mathrm{~b}$ \\
\hline
\end{tabular}

${ }^{\mathrm{y}}$ Infected tea leaves were assessed 15 days after inoculation by counting number of disease leaves, compared with the total leaves $(n=20)$. The experiment was conducted twice. NS $=$ no symptoms.

${ }^{z}$ Virulence of the isolates was evaluated by measuring the diameter of the typical necrotic lesion on infected tea leaves 7 days after wound or drop inoculation. The mean standard deviation was calculated for three replicates. Data within columns followed by same letter do not differ significantly according to Tukey's honestly significant difference test $(P \leq 0.05)$. 
provide the best resolution for resolving the Pestalotiopsis-like species as compared with any single marker.

In this study, cultural and conidial morphology, multigene sequence data, and pathogenicity tests were combined to confirm that Pseudopestalotiopsis camelliae-sinensis, $N$. clavispora, and Pestalotiopsis camelliae are all causal agents of tea gray blight, which suggests that this disease may be caused by a species complex. Of the three species, Pseudopestalotiopsis camelliae-sinensis and Pestalotiopsis camelliae are new species recently reported on $C$. sinensis in several tea-growing countries (Chen et al. 2017b; Liu et al. 2017; Maharachchikumbura et al. 2014). This study reports, for the first time, the characteristics of Pseudopestalotiopsis camelliaesinensis, N. clavispora, and Pestalotiopsis camelliae causing gray blight on $C$. sinensis in China. The phylogenetic relationships provided more extensive knowledge about the genomic organization in Pestalotiopsis-like species responsible for gray blight of tea.

Results of pathogenicity tests in this study showed that symptoms were not usually observed on nonwounded leaves when a conidial suspension was inoculated onto tea leaves (Table 4), suggesting that wounding may be necessary for symptom development. However, in some cases, wounding was not necessary for disease development on ginger and lychee but symptoms were enhanced by wounds (Keith et al. 2006). Further studies on the pathogenic mechanism of pathogens causing tea gray blight disease are underway.

\section{Acknowledgments}

This work was supported by the National Natural Science Foundation of China (NSFC) (31700607), the Natural Science Foundation Project of CQ CSTC (cstc2015jcyjA80019) and the Fundamental Research Funds for the Central Universities (XDJK2017C045 and XDJK2017D118) for financial support.

\section{Literature Cited}

Agnihothrudu, V. 1964. A world list of fungi reported on tea. J. Madras Univ. 34: $155-271$

Barr, M. E. 1975. Pestalosphaeria, a new genus in the Amphisphaeriaceae. Mycologia 67:187-194.

Barr, M. E. 1990. Prodromus to nonlichenized, pyrenomycetous members of class Hymenoascomycetes. Mycotaxon 39:43-184.

Carbone, I., and Kohn, L. M. 1999. A method for designing primer sets for speciation studies in filamentous ascomycetes. Mycologia 91:553-556.

Chen, Y. J., Qiao, W. J., Zeng, L., Shen, D. H., Liu, Z., Wang, S. H., and Tong, H. R. 2017a. Characterization, pathogenicity and phylogenetic analyses of Colletotrichum species associated with Brown Blight Disease on Camellia sinensis in China. Plant Dis. 101:1022-1028.

Chen, Y. J., Zeng, L., Shu, N., Wang, H., and Tong, H. R. 2017b. First report of Pestalotiopsis camelliae causing grey blight disease on Camellia sinensis in China. Plant Dis. 101:1034.

Crous, P. W., Summerell, B. A., Swart, L., Denman, S., Taylor, J. E., Bezuidenhout, C. M., Palm, M. E., Marincowitz, S., and Groenewald, J. Z. 2011. Fungal pathogens of Proteaceae. Persoonia 27:20-45.

Guerber, J. C., Liu, B., Correll, J. C., and Johnston, P. R. 2003. Characterization of diversity in Colletotrichum acutatum sensu lato by sequence analysis of two gene introns, mtDNA and intron RFLPs, and mating compatibility. Mycologia 95:872-895.

Hopkins, K. E., and McQuilken, M. P. 2000. Characteristics of Pestalotiopsis associated with hardy ornamental plants in the UK. Eur. J. Plant Pathol. 106: $77-85$

Horikawa, T. 1986. Yield loss of new tea shoots due to grey blight caused by Pestalotia longiseta Spegazzini. Bull. Shizuoka Tea Exp. Stn. 12:1-8.

Hu, H. L., Jeewon, R., Zhou, D. Q., Zhou, T. X., and Hyde, K. D. 2007. Phylogenetic diversity of endophytic Pestalotiopsis species in Pinus armandii and Ribes spp.: Evidence from rDNA and $\beta$ - tubulin gene phylogenies. Fungal Divers. 24:1-22.

Jeewon, R., Liew, E. C. Y., Simpson, J. A., Hodgkiss, I. J., and Hyde, K. D. 2003. Phylogenetic significance of morphological characters in the taxonomy of Pestalotiopsis species. Mol. Phylogenet. Evol. 27:372-383.

Joshi, S. D., Sanjay, R., Baby, U. I., and Mandal, A. K. A. 2009. Molecular characterization of Pestalotiopsis spp. associated with tea (Camellia sinensis) in southern India using RAPD and ISSR markers. Indian J. Biotechnol. 8: 377-383.

Kang, J. C., Hyde, K. D., and Kong, R. Y. C. 1999. Studies on the Amphisphaeriales: The Amphisphaeriaceae (sensu stricto). Mycol. Res. 103: $53-64$
Keith, L. M., Velasquez, M. E., and Zee, F. T. 2006. Identification and characterization of Pestalotiopsis spp. causing scab disease of guava, Psidium guajava in Hawaii. Plant Dis. 90:16-23.

Lateef, A. A., Sepiah, M., and Bolhassan, M. H. 2015. Description of Pseudopestalotiopsis kubahensis sp. nov., a new species of microfungi from Kubah National Park, Sarawak, Malaysia. Curr. Res. Environ. Appl. Mycol. 5:376-381

Lee, S., Crous, P. W., and Wingfield, M. J. 2006. Pestalotioid fungi from Restionaceae in the Cape Floral Kingdom. Stud. Mycol. 55:175-187.

Lin, Q., Kanchana-udomkan, C., Jaunet, T., and Mongkolporn, O. 2002. Genetic analysis of resistance to pepper anthracnose caused by Colletotrichum capsici. Thai J. Agric. Sci. 35:259-264.

Liu, A. R., Chen, S. C., Wu, S. Y., Xu, T., Guo, L. D., Jeewon, R., and Wei, J. G. 2010. Cultural studies coupled with DNA based sequence analyses and its implication on pigmentation as a phylogenetic marker in Pestalotiopsis taxonomy. Mol. Phylogenet. Evol. 57:528-535.

Liu, F., Hou, L., Raza, M., and Cai, L. 2017. Pestalotiopsis and allied genera from Camellia, with description of 11 new species from China. Sci. Rep. 7: Article 866. doi:10.1038/s41598-017-00972-5

Maharachchikumbura, S. S. N., Chukeatirote, E., Guo, L. D., Crous, P. W., McKenzie, E. H. C., and Hyde, K. D. 2013a. Pestalotiopsis species associated with Camellia sinensis (tea). Mycotaxon 123:47-61.

Maharachchikumbura, S. S. N., Guo, L. D., Cai, L., Chukeatirote, E., Wu, W. P., Sun, X., Crous, P. W., Bhat, D. J., McKenzie, E. H. C., Bahkali, A. H., and Hyde, K. D. 2012. A multi-locus backbone tree for Pestalotiopsis, with a polyphasic characterization of 14 new species. Fungal Divers. 56:95-129.

Maharachchikumbura, S. S. N., Guo, L. D., Chukeatirote, E., Bahkali, A. H., and Hyde, K. D. 2011. Pestalotiopsis-Morphology, phylogeny, biochemistry and diversity. Fungal Divers. 50:167-187.

Maharachchikumbura, S. S. N., Guo, L. D., Chukeatirote, E., and Hyde, K. D. 2013b. Improving the backbone tree for the genus Pestalotiopsis; addition of $P$. steyaertii and P. magna sp. nov. Mycol. Prog. 13:617-624.

Maharachchikumbura, S. S. N., Guo, L. D., Liu, Z. Y., and Hyde, K. D. 2016 Pseudopestalotiopsis ignota and Ps. camelliae spp. nov. associated with grey blight disease of tea in China. Mycol. Prog. 15:22.

Maharachchikumbura, S. S. N., Hyde, K. D., Groenewald, J. Z., Xu, J., and Crous, P. W. 2014. Pestalotiopsis revisited. Stud. Mycol. 79:121-186.

Muraleedharan, N., and Chen, Z. M. 1997. Pests and diseases of tea and their management. J. Plant Crop 25:15-43.

O’Donnell, K., and Cigelnik, E. 1997. Two divergent intragenomic rDNA ITS2 types within a monophyletic lineage of the fungus Fusarium are nonorthologous. Mol. Phylogenet. Evol. 7:103-116.

Pirone, P. P. 1978. Diseases and Pests of Ornamental Plants. Wiley Interscience, New York.

Rehner, S. A. 2001. Primers for elongation factor 1-alpha (EF1-alpha). https://web archive.org/web/20080514151555/http://ocid.nacse.org/research/deephyphae/ EF1primer.pdf

Saitou, N., and Nei, M. 1987. The neighbor-joining method: A new method for reconstructing phylogenetic trees. Mol. Biol. Evol. 4:406-425.

Sanjay, R., Ponmurugan, P., and Baby, U. I. 2008. Evaluation of fungicides and biocontrol agents against grey blight disease of tea in the field. Crop Prot. 27:689-694

Sutton, B. C. 1980. The Coelomycetes. Fungi Imperfecti with Pycnidia, Acervuli and Stromata. Commonwealth Mycological Institute, Kew, Surrey, UK.

Takeda, Y. 2002. Genetic analysis of tea gray blight resistance in tea plants. JARQ 36:143-150.

Tamura, K., Stecher, G., Peterson, D., and Lewis, P. O. 2013. MEGA6: Molecular Evolutionary Genetics Analysis version 6.0. Mol. Biol. Evol. 30:2725-2729.

Than, P. P., Jeewon, R., Hyde, K. D., Pongsupasamit, S., Mongkolporn, O., and Taylor, P. W. J. 2008. Characterization and pathogenicity of Colletotrichum species associated with anthracnose on chilli (Capsicum spp.) in Thailand. Plant Pathol. 57:562-572.

Wei, J. G., Xu, T., Guo, L. D., Liu, A. R., Zhang, Y., and Pan, X. H. 2007 Endophytic Pestalotiopsis species associated with plants of Podocarpaceae, Theaceae and Taxaceae in southern China. Fungal Divers. 24:55-74.

White, T. J., Bruns, T., Lee, S., and Taylor, J. 1990. Amplification and direct sequencing of fungal ribosomal RNA genes for phylogenetics. Pages 315-322 in: PCR Protocols: A Guide to Methods and Amplifications. M. A. Innis, D. H. Gelfand, J. J. Sninsky, and T. J. White, eds. Academic Press, San Diego, CA.

Zhang, Y. M., Maharachchikumbura, S. S. N., McKenzie, E. H. C., and Hyde, K. D. 2012a. A novel species of Pestalotiopsis causing leaf spots of Trachycarpus fortunei. Cryptogam. Mycol. 33:322-318.

Zhang, Y. M., Maharachchikumbura, S. S. N., Tian, Q., and Hyde, K. D. 2013. Pestalotiopsis species on ornamental plants in Yunnan Province, China. Sydowia 65:59-74.

Zhang, Y. M., Maharachchikumbura, S. S. N., Wei, J. G., McKenzie, E. H. C., and Hyde, K. D. 2012b. Pestalotiopsis camelliae, a new species associated with grey blight of Camellia japonica in China. Sydowia 64:335-344. 